\title{
Mechanism of Desynchronization in the Finite-Dimensional Kuramoto Model
}

\author{
Yu. Maistrenko, ${ }^{1,2,4}$ O. Popovych, ${ }^{1,4}$ O. Burylko, ${ }^{4}$ and P. A. Tass ${ }^{1,3}$ \\ ${ }^{1}$ Institute of Medicine, Research Center Jülich, 52425 Jülich, Germany \\ ${ }^{2}$ Central Institute for Electronics, Research Center Jülich, 52425 Jülich, Germany \\ ${ }^{3}$ Department of Stereotaxic and Functional Neurosurgery, University Hospital, 50924 Cologne, Germany \\ ${ }^{4}$ Institute of Mathematics, National Academy of Sciences of Ukraine, 01601 Kyiv, Ukraine
}

(Received 18 December 2003; published 19 August 2004)

\begin{abstract}
We study how a decrease of the coupling strength causes a desynchronization in the Kuramoto model of $N$ globally coupled phase oscillators. We show that, if the natural frequencies are distributed uniformly or close to that, the synchronized state can robustly split into any number of phase clusters with different average frequencies, even culminating in complete desynchronization. In the simplest case of $N=3$ phase oscillators, the course of the splitting is controlled by a Cherry flow. The general $N$-dimensional desynchronization mechanism is numerically illustrated for $N=5$.
\end{abstract}

DOI: 10.1103/PhysRevLett.93.084102

Starting with the work of Winfree [1] and Kuramoto [2], there has been growing interest in synchronization of globally coupled limit cycle oscillators [3-8]. Possible applications include many self-organizing systems in physics, chemistry, biology, and medicine and range, e.g., from Josephson junction arrays [9], semiconductor lasers arrays [10], chemistry [11], cardiac pacemaker cells [12], and flashing fireflies [13] to the development of demand-controlled brain pacemakers for the therapy of neurological and psychiatric diseases [14,15]. Fundamental to all such applications is to understand mechanisms that cause synchronization or desynchronization.

If the coupling between limit cycle oscillators is strong enough, in general, phase synchronization occurs: All oscillators rotate with the same average frequency. With decreasing coupling strength, desynchronization occurs: The oscillators split into groups of different average frequencies, such that inside each group the frequency is the same. This transition is called a frequency-splitting bifurcation. It is a common property of very different ensembles of coupled oscillators with both local and global coupling, characterized by regular or chaotic dynamics (see, e.g., [16-19], and references therein).

General properties of the phase dynamics in ensembles of nonlinear limit cycle oscillators with weak global coupling are described by the Kuramoto model [2]

$$
\dot{\psi}_{i}=\omega_{i}+\frac{K}{N} \sum_{j=1}^{N} \sin \left(\psi_{j}-\psi_{i}\right), \quad i=1, \ldots, N,
$$

where $\psi_{i}$ are phase variables, $\omega_{i}$ are natural frequencies, and $K>0$ is a coupling parameter. In the thermodynamic limit $N \rightarrow \infty$, a transition from the synchronized state to a complete desynchronization occurs, when the strength of the coupling decreases below a certain critical value. In spite of numerous studies, in the finite-dimensional Kuramoto model the desynchronization mechanism is still far from being well understood (see, e.g., [17], and references therein). This issue is the starting point of the present Letter. Unlike Kuramoto, Strogatz, and others
PACS numbers: $05.45 . \mathrm{Xt}$

$[2,17]$, we do not use well-developed statistical techniques. Rather, we here use a qualitatively different approach, which is based on bifurcation theory.

In system (1), the regime of complete synchronization is destroyed when the coupling strength decreases below $K=K_{c}$ giving rise to an appearance of phase clusters. In most cases, at the bifurcation, only one of the phase variables $\psi_{i}$ splits off from the others, and thus only two phase clusters arise, which contain 1 and $N-1$ elements [see Fig. 1(a)]. This codimension one bifurcation is well known for the unimodal (Gaussian) distributions of the natural frequencies $\omega_{i}$ in the Kuramoto model [16].

The purpose of this Letter is to show that if the natural frequencies in the Kuramoto model are distributed uniformly, or close to that, the number of the phase clusters
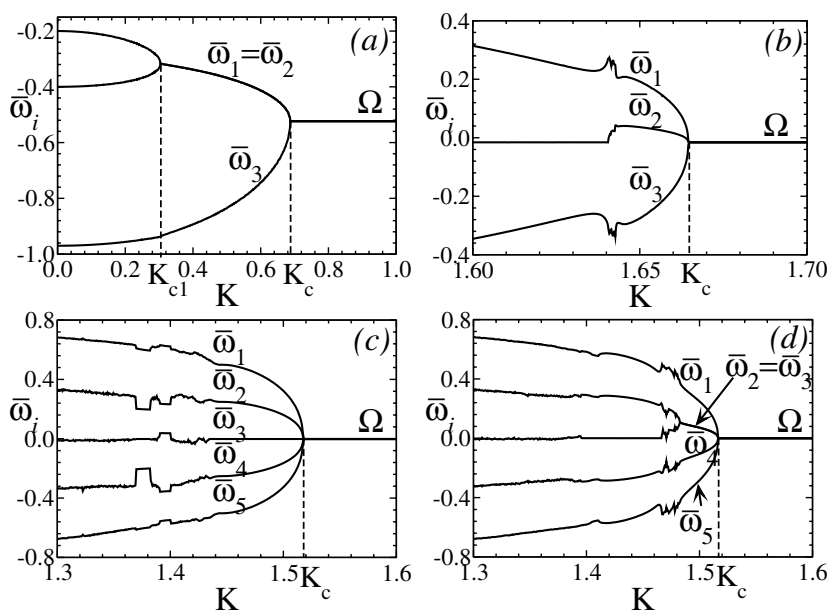

FIG. 1. Frequency-splitting bifurcation diagrams for the Kuramoto model from Eq. (1) with $N=3$ [(a),(b)] and $N=5[$ (c),(d)] phase oscillators, with a nonuniform distribution of the natural frequencies $\omega_{i}$ : (a) $\{-0.97,-0.4,-0.2\}$, (b) $\{-1.0,0.0,0.953\}, \quad$ (c) $\{-1.0022,-0.50446,0.00106$, $0.49869,0.99804\}$, and (d) $\{-1.00064,-0.50017,0.00347$, $0.50170,0.99852\} . \bar{\omega}_{i}=\left\langle\dot{\psi}_{i}\right\rangle$, where $\langle\cdot\rangle$ denotes averaging in time. $\Omega$ is the mean frequency. 
instantly emerging due to the frequency-splitting bifurcation at $K=K_{c}$ may also be three and more, ranging even up to complete desynchronization [see Fig. 1(b)1(d)]. Moreover, our results show that the frequencysplitting bifurcation is also of codimension one in the case where it generates multiple clusters. Thus small parameter perturbations of the Kuramoto model do not destroy the course of the desynchronization transition.

Based on the theory of low-dimensional dynamical systems, we unfold the mechanism of the frequencysplitting bifurcation by considering the simplest, nontrivial case of only $N=3$ coupled oscillators in the Kuramoto model (1). Then, by introducing phase difference variables $\psi_{2}-\psi_{1}$ and $\psi_{3}-\psi_{2}$, the problems is reduced to a flow on a two-dimensional torus, which is a so-called Cherry flow [20-24]. Following Ref. [23], a Cherry flow is defined as a smooth flow on a twodimensional torus, which has two equilibria, one being a saddle and the other being a sink or source, and rotating trajectories of some rotation number which is called the winding ratio. For the Cherry flow obtained for the Kuramoto model, the second equilibrium is a stable node and torus rotations are unstable. During the frequency-splitting bifurcation, the two equilibria disappear in a saddle-node bifurcation, and the preexisting unstable Cherry flow rotations capture the whole torus volume, where they inherit the winding ratio. Then, in the bifurcation moment $K=K_{c}$, zero Cherry flow winding ratio induces cluster doubling; nonzero one causes cluster tripling.

Figure 1 illustrates different types of the frequencysplitting bifurcations of the Kuramoto model with $N=3$ and $N=5$. In Fig. 1(a), a standard bifurcation sequence is plotted. With decreasing $K$, first, at $K=K_{c}$, complete synchronization $\bar{\omega}_{1}=\bar{\omega}_{2}=\bar{\omega}_{3}=\Omega$ turns into two clusters with unequal frequencies $\bar{\omega}_{1}=\bar{\omega}_{2}$ and $\bar{\omega}_{3}$. Then, at a smaller coupling parameter value $K_{c 1}$, the first cluster $\bar{\omega}_{1}=\bar{\omega}_{2}$ splits, in turn, causing a complete desynchronization: $\bar{\omega}_{1} \neq \bar{\omega}_{2} \neq \bar{\omega}_{3}$. Both bifurcations may be referred to as cluster doubling. In Fig. 1(b) an example of a cluster tripling is presented. The synchronous motion breaks up at $K=K_{c}$, instantly producing three different frequencies. Furthermore, just after the bifurcation, successive frequency differences $\bar{\Delta}_{1}=\bar{\omega}_{2}-\bar{\omega}_{1}$ and $\bar{\Delta}_{2}=$ $\bar{\omega}_{3}-\bar{\omega}_{2}$ become locked in a 1:2 resonance: $\bar{\Delta}_{1} / \bar{\Delta}_{2}=$ $1: 2$. Below we will show that, depending on the parameters, at the bifurcation any other resonance $p: q$ can be obtained in a robust way. Figures 1(c) and 1(d) refer to a five-dimensional Kuramoto model, in which with decreasing coupling strength the common average frequency $\Omega$ instantly splits into five and four clusters, respectively. The 1:1:1:1 resonance [Fig. 1(c)] and the 1:1:0:1 resonance [Fig. 1(d)] take place for the fourdimensional torus dynamics of the corresponding phase differences. Irregular behavior in Figs. 1(b)-1(d) corresponds to the transition through narrow resonance tongues of different winding ratios as coupling decreases.
To describe the mechanism of the frequency-splitting bifurcation, consider in detail the Kuramoto model (1) for $N=3$. It can easily be reduced to the two-dimensional system of the phase differences $\varphi_{1}=\psi_{2}-\psi_{1}$ and $\varphi_{2}=$ $\psi_{3}-\psi_{2}$ :

$$
\begin{aligned}
& \dot{\varphi}_{1}=\Delta_{1}+\frac{K}{3}\left[\sin \left(\varphi_{2}\right)-\sin \left(\varphi_{1}+\varphi_{2}\right)-2 \sin \left(\varphi_{1}\right)\right], \\
& \dot{\varphi}_{2}=\Delta_{2}+\frac{K}{3}\left[\sin \left(\varphi_{1}\right)-\sin \left(\varphi_{1}+\varphi_{2}\right)-2 \sin \left(\varphi_{2}\right)\right],
\end{aligned}
$$

where $\Delta_{1}=\omega_{2}-\omega_{1}$ and $\Delta_{2}=\omega_{3}-\omega_{2}$. Consider the flow $\Phi$ defined by Eq. (2), which is a flow on a twodimensional torus $\mathbb{T}^{2}=[0 ; 2 \pi]^{2}$.

Let first $\Delta_{1}=\Delta_{2}=0$. In that case, complete synchronization takes place. The flow $\Phi$ possesses six equilibria, each characterized by two Lyapunov exponents $\lambda_{1}$ and $\lambda_{2}$ : a sink at the origin $O(0,0)$, with $\lambda_{1}=\lambda_{2}=-K$; three saddles $S_{1}(0, \pi), S_{2}(\pi, 0)$, and $S_{3}(\pi, \pi)$, with $\lambda_{1}=K$ and $\lambda_{2}=-K / 3$; and two sources $N_{1}(2 \pi / 3,2 \pi / 3)$ and $N_{2}(4 \pi / 3,4 \pi / 3)$, with $\lambda_{1}=\lambda_{2}=K / 2$ [Fig. 2(a)]. Stable manifolds of the saddles play the role of "stoppers" for the torus dynamics. Any trajectory of system (2) originating anywhere (except for the unstable equilibria and the stable manifolds of the saddles) approaches the sink $O$ without even a single rotation around the torus.

Let now $\Delta_{1}=\Delta_{2} \stackrel{\text { def }}{=} \Delta>0$. Then, system (2) preserves its symmetry with respect to the diagonal $D\left(\varphi_{1}=\varphi_{2}\right)$, which is an invariant manifold of the torus flow $\Phi$, and Eq. (2) on it turns into

$$
\dot{\varphi}=\Delta-\frac{K}{3}[\sin (\varphi)+\sin (2 \varphi)],
$$

where $\varphi \stackrel{\text { def }}{=} \varphi_{1}=\varphi_{2}$. For the values of $\Delta$ that are positive and small enough, Eq. (3) has four equilibria $O, N_{1}, S_{2}$, and $N_{2}$. With decreasing control parameter $K / \Delta$, first, $S_{2}$ and $N_{2}$ annihilate in an inverse saddle-node bifurcation at $K \approx 8.13 \Delta$. Then, at $K=3 \Delta$ a pitchfork bifurcation occurs, where the out of the diagonal saddles $S_{1}$ and $S_{3}$
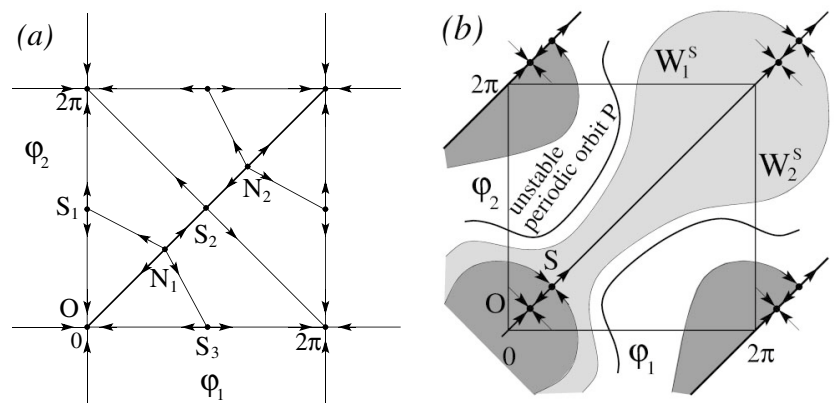

FIG. 2. Schematic representation of the phase portraits for Eq. (2) with $\Delta_{1}=\Delta_{2}=\Delta$. (a) $\Delta=0$ : The phase portrait contains a stable node $O$, saddles $S_{1}, S_{2}$, and $S_{3}$, and unstable nodes $N_{1}$ and $N_{2}$. (b) $\Delta>0$ and $1.70<K / \Delta<3$ (Cherry flow): The phase portrait contains a stable node $O$, a saddle $S$, and an unstable periodic orbit $P$, and is foliated by stable manifolds $W_{1}^{S}$ and $W_{2}^{S}$ of the saddle $S$, as indicated by the gray regions. 
collide with the unstable node $N_{1}$, in this way producing, instead, a saddle $S$.

The torus flow obtained after the pitchfork bifurcation is an example of a Cherry flow [20-24] [see Fig. 2(b)]. It contains two equilibria, sink $O$ and saddle $S$, and an unstable periodic orbit $P$. In the considered symmetrical case, obviously, the winding ratio of the Cherry flow equals $1: 1$. Indeed, along the orbit $P$, the torus variables $\varphi_{1}$ and $\varphi_{2}$ are locked in a 1:1 resonance. All other trajectories, except for $P$, saddle $S$, and its stable manifolds $W_{1,2}^{S}$ are attracted by the steady state $O$. Complete synchronization is not yet destroyed.

With further decreasing coupling parameter $K$, the frequency-splitting bifurcation occurs at $K \approx 1.70 \Delta$. The stable node $O$ and the saddle $S$ vanish due to a saddle-node bifurcation producing a stable periodic orbit. In the considered symmetrical case, this reemerging stable periodic orbit coincides with the diagonal $D$. All trajectories of Eq. (2) now rotate around the torus, and their winding ratio is $1: 1$, as before. Hence, both phase differences, $\varphi_{1}=\psi_{2}-\psi_{1}$ and $\varphi_{2}=\psi_{3}-\psi_{2}$, start to grow, which corresponds to a desynchronization in the original Kuramoto system in form of a cluster tripling.

The bifurcation properties obtained above for the case of equal natural frequency differences are preserved, in general, also if the differences $\Delta_{1}$ and $\Delta_{2}$ are different. Figure 3(a) presents the bifurcation diagram in the parameter plane $K / \Delta_{2}$ versus $\Delta_{1} / \Delta_{2}$. (Without loss of generality, the case $0<\Delta_{1} \leq \Delta_{2}$ is considered.) The three saddle-node bifurcation curves $B_{1}, B_{2}$, and $B_{c}$ correspond to pairwise saddle-node annihilation of the six equilibria of Eq. (2). Above the middle bifurcation curve $B_{2}$, the torus flow $\Phi$ remains closed (without any rotations). The Cherry flow exists in the hatched parameter region between the $B_{2}$ and $B_{c}$ curves. Its winding ratio varies continuously with the parameters as a devil's staircase and may be rational or irrational. In Fig. 3(a) two main resonant tongues are shown, with winding ratio 0:1 (light gray) and 1:1 (dark gray). The tongues emanate from the corner parameter points 0 and 1 of the $K=0$ axis, respectively, and end touching each other in the singular point $C=(1,3)$. All other resonant tongues, with wind-
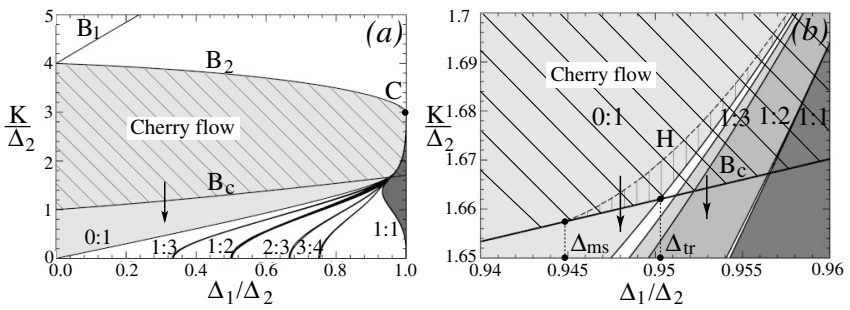

FIG. 3. (a) Two-parameter bifurcation diagram for Eq. (1) with $N=3$. The Cherry flow region is hatched. The main resonant tongues $0: 1,1: 3,1: 2,2: 3,3: 4$, and $1: 1$ are shown. (b) Enlargement from (a). Parameter variations along the arrows in (a) and (b) correspond to the bifurcation diagrams in Figs. 1(a) and 1(b), respectively. ing ratios $p: q$, originate from the points $p / q$ in the $K=0$ axis and are aligned in between the 0:1 and 1:1 resonant tongues. Also, they all extend up to the singular parameter point $C(1,3)$, where they glue together and end. Quasiperiodic torus rotations take place in the fractal parameter set which complements the union of the resonant tongues. This fractal parameter set appears to be very thin: both the Lebesgue measure and the Hausdorff dimension of it equal zero (see Refs. [21,22]). Hence, the probability of observing a quasiperiodic Cherry flow is negligible.

Figure 3(b) presents an enlargement of the bifurcation diagram given by Fig. 3(a) for the region where the $B_{c}$ bifurcation curve intersects the resonant tongues. The enlargement shows that the resonant tongues that exist above $B_{c}$ are naturally continued in the desynchronization region below $B_{c}$. The widest tongue has a $0: 1$ winding ratio, which means that inside it only the second torus variable $\varphi_{2}$ rotates; i.e., only the second phase difference $\psi_{3}-\psi_{2}$ starts to grow when the parameter point intersects $B_{c}$. Consequently, in that case, the frequencysplitting bifurcation gives rise to two phase clusters only, containing one and two oscillators, respectively [see Fig. 1(a), where the parameter varies as indicated by the arrow in Fig. 3(a)]. Such a type of cluster doubling takes place for all $\Delta_{1} / \Delta_{2}<\Delta_{\text {tr }}$, where $\Delta_{\text {tr }} \approx 0.95026$ is an intersection point of the 0:1 tongue boundary with the $B_{c}$ bifurcation curve. At other parameter values, i.e., for $\Delta_{1} / \Delta_{2}>\Delta_{\text {tr }}$, the winding ratio of the Cherry flow does not vanish, and thus the desynchronization transition always shows up as a cluster tripling: Both phase differences $\varphi_{1}$ and $\varphi_{2}$ start growing, in this way causing an instant splitting of all three phase variables $\psi_{1}, \psi_{2}$, and $\psi_{3}$. This transition is illustrated in Fig. 1(b), where the parameters vary as indicated by the right arrow in Fig. 3(b).

The desynchronization transition shown in Fig. 3 appears to be more complicated if it happens near the boundaries of the Cherry flow resonant tongues. Indeed, as we have found, the resonant tongues contain thin boundary layer strips, where a stable periodic orbit exists (see [24]), which is born in a homoclinic bifurcation. In Fig. 3(b) the homoclinic bifurcation curve $H$ is shown as a dashed line inside the 0:1 tongue. [Analogous homoclinic bifurcation curves giving rise to a stable periodic orbit can be found inside all other resonant tongues. But they are so thin that they cannot be resolved with the given scale in Fig. 3(b)]. Therefore, two attracting states coexist in the sideband, i.e., the vertically hatched region between the homoclinic bifurcation curve $H$ and the tongue boundary: (i) the Cherry flow steady state $O$ and (ii) the stable periodic orbit emerging in the homoclinic bifurcation at $H$. As our calculations show, this sideband multistability region inside the 0:1-resonant tongue is attached to the $B_{c}$ saddle-node curve, comprising an interval $\Delta_{\mathrm{ms}}<\Delta_{1} / \Delta_{2}<\Delta_{\mathrm{tr}}$. Then, if the desynchronization transition goes on in this interval [e.g., when $K / \Delta_{2}$ is decreased along the left arrow in Fig. 3(b)], synchronization 


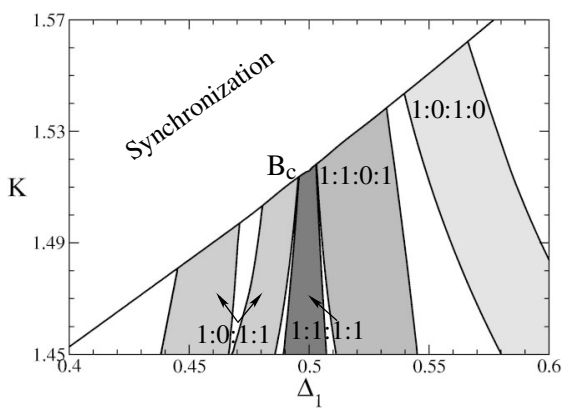

FIG. 4. Two-parameter bifurcation diagram for the Kuramoto model from Eq. (1) with $N=5$ and $\Delta_{i}=0.5, i=$ $\overline{2,4}$. Four main resonant tongues are shown.

and desynchronization coexist for the range of parameters between $H$ and $B_{c}$. These sideband multistability regions extend along the boundaries of the Cherry flow resonant tongues up to the singular parameter point $C(1 ; 3)$. Therefore, the desynchronizing behavior in the Kuramoto model can actually arise before the disappearance of the stable phase-locked state.

We expect that this low-dimensional mechanism also controls the frequency-splitting bifurcation in the general $N$-dimensional Kuramoto model from Eq. (1). In this case, the dynamics is reduced to an $(N-1)$-dimensional torus flow of the phase differences $\varphi_{i}=\psi_{i+1}-\psi_{i}$, which is an $(N-1)$-dimensional analog of Eq. (2). For $K>K_{c}$, synchronization is caused, generally, by a unique sink $O$. When $K$ decreases to $K_{c}$, this sink and, thus, also synchronization vanish due to an inverse saddle-node bifurcation. For a range of the coupling parameter values greater than $K_{c}$, some of the phase differences $\varphi_{i}$ already display circular rotations on the $(N-1)$-dimensional torus (such as for the Cherry flow in dimension two). Let us characterize these rotations by the $(N-1)$-dimensional analog of the winding ratio, $r_{1}: \cdots: r_{N-1}$, which indicates how many phase differences $\varphi_{i}$ rotate, and with which ratio with respect to each other. When the steady state $O$ disappears at $K=K_{c}$, the stable torus rotations arise, in this way causing a desynchronization. As in the threedimensional case, we expect the number of phase clusters emerging in the $N$-dimensional frequency-splitting bifurcation to be equal to the number of nonzero coordinates of the corresponding $(N-1)$-dimensional winding ratio +1 . This mechanism is robust with respect to small parameter perturbations. We conclude that the frequency-splitting bifurcation in the Kuramoto model, in the case where more than two phase clusters emerge, is also of codimension one.

Figure 4 illustrates this phenomenon in the case of the Kuramoto model from Eq. (1) with $N=5$ : Four main resonant tongues $1: 1: 1: 1,1: 0: 1: 1,1: 1: 0: 1$, and 1:0:1:0 are shown. The tongues hit the frequency-splitting bifurcation curve $B_{c}$. Our results obtained for $N=3$ suggest that the tongues continue above the $B_{c}$ curve in terms of a four-dimensional analog of the Cherry flow. However, for $N>3$, a bifurcation analysis is essentially more complicated and requires additional studies. The analysis gets more involved due to the appearance of complex dynamics and chaos in high-dimensional systems. For the Kuramoto model, chaos occurs beginning from the dimension $N=4$, as we shall present in a forthcoming study.

We greatly acknowledge fruitful and illuminating discussions with B. Fiedler, A. Pikovsky, L. P. Shilnikov, S. Yanchuk, and M. Zaks.

[1] A.T. Winfree, The Geometry of Biological Times (Springer, New York, 1980).

[2] Y. Kuramoto, Chemical Oscillations, Waves and Turbulence (Springer-Verlag, Berlin, 1984).

[3] G. B. Ermentrout, Physica (Amsterdam) 41D, 219 (1990).

[4] H. Daido, Int. J. Bifurcation Chaos Appl. Sci. Eng. 7, 807 (1997), and references therein.

[5] L. Ren and B. Ermentrout, Physica (Amsterdam) 143D, 56 (2000).

[6] J. T. Ariaratnam and S. H. Strogatz, Phys. Rev. Lett. 86, 4278 (2001).

[7] J. A. Acebron, A. Perales, and R. Spigler, Phys. Rev. E 64, 016218 (2001)

[8] E. Ott, P. So, E. Barreto, and T. Antonsen, Physica (Amsterdam) 173D, 29 (2002).

[9] K. Wiesenfeld, P. Colet, and S. H. Strogatz, Phys. Rev. Lett. 76, 404 (1996); Phys. Rev. E 57, 1563 (1998).

[10] G. Kozyreff, A. G. Vladimirov, and P. Mandel, Phys. Rev. Lett. 85, 3809 (2000).

[11] W. Wang, I. Z. Kiss, and J. L. Hudson, Chaos 10, 248 (2000); Phys. Rev. Lett. 86, 4954 (2001).

[12] C. S. Peskin, Mathematical Aspects of Heart Physiology (Courant Institute of Mathematical Sciences, New York, 1975).

[13] J. Buck, Q. Rev. Biol. 63, 265 (1988).

[14] P. A. Tass, Phase Resetting in Medicine and Biology (Springer, Berlin, 1999).

[15] P. A. Tass, Europhys. Lett. 53, 15 (2001); Biol. Cybern. 87, 102 (2002); Phys. Rev. E 66, 036226 (2002).

[16] G. B. Ermentrout and N. Kopell, SIAM J. Math. Anal. 15, 215 (1984).

[17] S. H. Strogatz, Physica (Amsterdam) 143D, 1 (2000).

[18] A. Pikovsky, M. Rosenblum, and J. Kurths, Synchronization, A Universal Concept in Nonlinear Sciences (Cambridge University Press, Cambridge, 2001); D. Topaj and A. Pikovsky, Physica (Amsterdam) 170D, 118 (2002).

[19] M. Zhan, Z. Zheng, G. Hu, and X. Peng, Phys. Rev. E 62, 3552 (2000); Z. Zheng, G. Hu, and B. Hu, Phys. Rev. E 62, 7501 (2000).

[20] T. M. Cherry, Proc. London Math. Soc. 44, 175 (1938).

[21] C. Boyd, Ergod. Theory Dyn. Syst. 5, 27 (1985).

[22] J. J. P. Veerman, Nonlinearity 2, 419 (1989).

[23] C. Baesens, J. Guckenheimer, S. Kim, and R. S. MacKay, Physica (Amsterdam) 49D, 387 (1991).

[24] M. A. Zaks, Physica (Amsterdam) 149D, 237 (2001). 